\title{
Victim blaming and slut shaming: when the victim is criminalized, not the sex offender
}

\author{
Victim blaming e slut shaming: quando si criminalizza la vittima \\ e non il sex offender
}

\section{Culpar a la víctima y avergonzarla: cuando se criminaliza a la víctima y no al delincuente sexual}

\author{
Aurelia V. Nemesu \\ Dottoressa Magistrale in Investigazione, Criminalità e Sicurezza Internazionale, Università degli Studi Internazionali di \\ Roma (UNINT), Italy
}

\begin{abstract}
This article aims at analysing the phenomenon of child abuse, the consequences that it may produce in the different evolutionary stages and the role it plays in the manifestation of aggressive behaviour in adulthood. Also, it examines the relationship between the different types of abuse suffered during childhood and the development of criminal phenomenologies such as serial killers, spree killers and mass murderers.
\end{abstract}

\section{RIASSUNTO}

Il presente articolo mira all'analisi del fenomeno del maltrattamento infantile, delle conseguenze che questo può produrre nei differenti stadi evolutivi e del ruolo che ricopre nella manifestazione di comportamenti aggressivi in età adulta. Inoltre, viene esaminata la relazione che intercorre tra le diverse tipologie di abuso subito durante l'infanzia e lo sviluppo di fenomenologie criminali quali serial killer, spree killer e mass murderer.

\section{RESUMEN}

El presente artículo tiene por objeto analizar el fenómeno del abuso infantil, las consecuencias que éste puede producir en las diferentes fases evolutivas y la función que desempeña en la manifestación de comportamientos agresivos en la edad adulta. Ademàs, se examina la relación que existe entre los diferentes tipos de abuso sufrido durante la infancia y el desarrollo de fenomenologías delictivas como serial killer.

\section{Il fenomeno di victim blaming e slut shaming}

Fin da bambini veniamo cresciuti con il pensiero che le "signorine" non debbano avere certi comportamenti e/o atteggiamenti; che un uomo che ha un numero di amanti elevato è un playboy e che, pure se ne parla in giro, "non fa peccato", mentre una donna con una libido piuttosto forte non può concedersi il lusso di cambiare partner e di non vergognarsene, senza aspettarsi di essere appellata come una "sgualdrina" o una donna dai "facili costumi". È proprio sulla base di tali pensieri che si formano e maturano fenomeni come quello del victim blaming e dello slut shaming.

Il victim blaming, stando alla definizione di Wikipedia, "consiste nel ritenere la vittima di un crimine, o di altre sventure, parzialmente o interamente responsabile di ciò che le è accaduto e spesso - nell'indurre la vittima stessa ad auto colpevolizzarsi. Un atteggiamento di victim blaming è anche connesso con l'ipotesi che si deve conoscere e accettare una supposta "natura umana" (che sarebbe maligna in questa visione, o tendente all'abuso, alla sopraffazione), e - conseguentemente - adeguarcisi anche a scapito dei propri desideri, opinioni e della propria libertà". Si può dunque affermare che il victim blaming sia quell'atteggiamento il quale suggerisce che la vittima, piuttosto che l'autore del reato, sia responsabile dell'aggressione. Tale fenomeno si verifica quando si presume che un individuo abbia fatto qualcosa per provocare la violenza con azioni, parole o attraverso il modo in cui era vestita in quel determinato momento. Esso risulta essere una delle ragioni principali per cui le vittime di violenza sessuale e domestica non denunciano le loro aggressioni temendo in un giudizio negativo che possa portarle a sperimentare una forma di vittimizzazione secondaria durante il processo, e, successivamente, forti pregiudizi nei loro confronti all'interno della società in cui vivono. Il termine "victim blaming" è stato usato per la prima volta nel 1976 dallo psicologo William Ryan nel libro "Incolpare la vittima" come risposta alle dichiarazioni di chi, in quel periodo, sosteneva che le cause della povertà di una certa fascia della popolazione erano da imputare unicamente al comportamento dei singoli individui. Il testo di Ryan risulta essere dunque una critica a quella mentalità, la quale ritiene che si debba incolpare i poveri per la loro condizione di povertà, gli impotenti per la loro impotenza e, in riferimento al contesto che stiamo trattando in questo articolo, le vittime per la loro condizione di vittima. "Da un punto di vista esterno, incolpare le vittime per la situazione in cui si trovano è un modo semplice per affrontare situazioni difficili e permette, ad 
esempio, di ignorare il problema in quanto è responsabilità della vittima trovare il modo di risolverlo o imparare a conviverci" (Ryan, 1976). Ciò che William Ryan ha affermato nel suo libro sulla struttura della società, in riferimento alle persone in condizione di povertà, può essere applicato anche alle vittime di reati, in particolare alle vittime di violenza domestica e crimini sessuali. Risulta importante anche quanto affermato da Lerner nella sua opera "The belief in a just world. A Fundamental Delusion" del 1980 "Il pensiero che una vittima di un reato sia in qualche modo la causa dell'evento stesso, per esempio a causa del suo comportamento deviante o provocatorio, è confortante poiché comporta il pensiero che se io non mi comporto così, non mi accadrà quel determinato evento. Incolpare la vittima della sua sorte, dunque, ci permette di sentirci liberati delle responsabilità e di alleviare la paura di diventare noi stessi una vittima". L'ipotesi del mondo giusto, o meglio, la credenza in un mondo giusto proposta da Lerner nel 1980, ci aiuta a comprendere il processo psicologico del "victim-blaming". Secondo Lerner, le persone hanno la tendenza a pensare che tutti ricevano ciò che si meritano, al fine di mantenere nella loro mente l'idea di vivere in un mondo giusto, in una società in cui un cattivo comportamento (o caratteristiche personali negative) sono "ricompensate" con incidenti e disgrazie, mentre i buoni comportamenti possono solo portare a risultati positivi.

Il victim-blaming non è un atteggiamento mentale innocuo e può essere un ostacolo per le vittime quando è il momento di chiedere aiuto (per esempio una vittima di stupro può decidere di non segnalare ciò che è successo per paura di essere giudicata e ritenuta responsabile dell'accaduto); inoltre ciò può portare a una mancanza di sostegno sociale ed anche ad una scadente assistenza legale e medica. Uno degli elementi che porta le vittime di aggressione sessuale ad essere poi considerate colpevoli di quanto loro accaduto è proprio la sensualità del loro aspetto fisico e dell'abbigliamento adottato al momento del fatto reato. Tale elemento porta al dilagarsi di quel fenomeno che prende il nome di "slut shaming". Esempio emblematico della gravità e delle conseguenze di tale fenomeno è il caso della cittadina irlandese di Cork, cittadina in cui la biancheria intima di una vittima di stupro è stata trasformata nella prova della consensualità del rapporto, o del fatto che la donna aveva istigato l'uomo con un abbigliamento che alludeva alla sua disponibilità sessuale. In entrambi i casi, l'indumento è stato strumentalizzato per trasformare la vittima in un oggetto sessuale disponibile agli occhi del suo aggressore e come prova del suo essere co-responsabile di quanto accaduto. Rifacendoci anche in questo caso alla definizione di Wikipedia, slut-shaming significa "svergognare la sgualdrina". Vuol dire in pratica utilizzare elementi relativi alla condotta sessuale di qualcuno, o che rimandano a essa, per svalutare tale persona e imporre un giudizio negativo. Fondamentale risulta, ai fini di una maggior comprensione del fenomeno, tenere a mente che la sessualità è un campo talmente vasto, che investe così tanti aspetti della persona, che anche lo slutshaming può prendere molte forme, consapevoli e inconsapevoli: può colpire una persona appoggiandosi al suo modo di vestire, alla sua salute, al suo aspetto fisico o al suo orientamento sessuale, alla sua storia relazionale o ai suoi hobby. Come affermato dall'autrice Leona Tanenbaum nel 2015:

"Lo slut-shaming è più dannoso dei semplici insulti - anche se essere insultate pubblicamente in sé può essere traumatico, come dimostrano i suicidi di ragazze vittime di tale fenomeno. Una volta che una ragazza o una donna viene considerata una "sgualdrina", può diventare anche bersaglio di violenza sessuale. E se viene stuprata, le può essere assegnata tale etichetta per giustificare il carnefice".

Per quanto la letteratura di ricerca sulle conseguenze di tali fenomeni (victim blaming e slut shaming) sia ancora abbastanza limitata, da queste prime evidenze si può capire come sia urgente indagare a fondo le conseguenze di fenomeni che sono in grado di incidere profondamente sia sulla vita delle persone che ne fanno esperienza, sia sul più ampio piano sociale e giuridico. Oltre alla constatazione che sia sbagliato incolpare la parte offesa, la quale avrebbe piuttosto bisogno di assistenza e non di giudizi dannosi, il victim blaming e lo slut shaming portano a quella che viene chiamata vittimizzazione secondaria.

Secondo il criminologo Lino Rossi (2005) la vittimizzazione secondaria rappresenta la condizione di ulteriore oltraggio e sofferenza sperimentata dalla vittima in relazione ad un atteggiamento di insufficiente attenzione o negligenza da parte delle agenzie di controllo formale nella fase del loro intervento e si manifesta nelle ulteriori conseguenze psicologiche sperimentate dalla vittima.

\section{L'impatto psicologico del crimine sulle vittime}

Di particolare rilevanza solo le possibili conseguenze psicologiche che possono scaturire a seguito di un'offesa, come la perdita del senso di controllo o il sentimento di violazione del sé. La memoria dell'offesa è di frequente destinata a protrarsi nel tempo, a volte come ricordo indelebile, e ciò dipende dalla gravità oggettiva del danno, ma ancora di più dalla capacità della vittima di reagire e dalle effettive risorse relazionali e sociali di cui essa dispone nel suo contesto di appartenenza. Quest'ultimo può non essere in grado di sostenere e aiutare la vittima come in realtà necessiterebbe, contribuendo così a rafforzare la vittimizzazione invece di limitarla (Vezzadini, 2012). L'impossibilità di cancellare l'offesa dal proprio percorso di vita, porta la persona a rispondere diversamente - da un punto di vista relazionare ed emozionale - all'accaduto, con una inevitabile messa in discussione della solidità e della centralità dei riferimenti valoriali e normativi oltre che dei rapporti che precedentemente riteneva fondamentali. La letteratura presa qui in esame si riferisce alle vittime di crimini di varia entità, con un'attenzione particolare alle vittime di stupro e di violenza domestica perpetrata dal partner, su cui si concentra la maggior parte degli studi a nostra disposizione. Dal punto di vista della psicologia, il crimine rappresenta per la vittima un agente stressante (stressor) traumatico, in grado di provocare reazioni psicopatologiche più o meno intense, in relazione alla sua dimensione oggettiva e soggettivamente percepita e alle capacità della vittima di fronteggiare ciò (coping). Uno stressor traumatico rappresenta una minaccia significativa, in grado di provocare reazioni di stress particolarmente debilitanti a cui è difficile far fronte. Nei casi più gravi, il trauma può determinare l'insorgenza del Disturbo Post-Traumatico da Stress (DPTS) che, secondo la quinta ed ultima edizione del Manuale diagnostico e statistico dei disturbi mentali risulta diagnosticabile dopo 30 giorni dal fatto traumatico. Il DPTS sostituisce i precedenti concetti di nevrosi traumatica o post-traumatica e i sintomi tipici di tale disturbo sono il rivivere l'esperienza traumatica tramite ad esempio sogni, flashback o ancora ricordi, una costante ipervigilanza, l'evitamento di stimoli che possono essere connessi al trauma, distacco emotivo dal contesto. Molti autori hanno sostenuto la possibilità di individuare alcune fasi comuni della reazione psicologica successiva alla vittimizzazione che una persona può subire.

Nello specifico abbiamo:

"La fase dell'impatto-disorganizzazione" individuato dagli studiosi Bard e Sangrey, 1986, la quale si manifesta immediatamente dopo la vittimizzazione subita dalla vittima. Quest'ultima finisce con il sperimentare una sorta di addormentamento, disorientamento insieme a sentimenti di negazione, incredulità, vulne- 
rabilità e senso di impotenza. Ciò comporta anche la presenza di ansia, di disturbo del sonno con possibilità di incubi e un possibile aggravamento di alcune problemi medici preesistenti;

"La fase del rifiuto" che si manifesta a breve termine dalla vittimizzazione, dunque dopo qualche ora oppure qualche giorno. Tali conseguenze hanno una durata media di circa 3-8 mesi. La vittima finisce con lo sperimentare emozioni che vanno dalla rabbia alla paura, dalla tristezza all'euforia e dall'autocommiserazione all'auto-colpevolizzazione. Con l'attenuarsi di tali emozioni, la vittima entra nella fase finale;

"La fase della riorganizzazione" in cui, se la vittima è in grado di affrontare e risolvere il trauma a cui è stata sottoposta, la vittimizzazione a questo punto si può convertire in una sorta di evoluzione personale. C'è da evidenziare, però, ciò molto spesso non avviene.

Possiamo dunque affermare che, le tre aree di indagine relative alle conseguenze subite dalle vittime di violenza sessuale sono:

- area psicologica;

- area relativa alla modificazione dello stile di vita della vittima; - area relativa alla modificazione dell'organizzazione dei rapporti interpersonali della vittima. Stando a una ricerca condotta dall'OMS (Organizzazione Mondiale della Sanità) è emerso che:

- Le donne, sia adolescenti che adulte, che hanno subito un abuso da parte del partner sono maggiormente esposte a depressione, ansia e fobie, pensieri e tentativi di suicidio rispetto alle donne che non hanno subito abusi.

- In uno studio di popolazione, la prevalenza dei sintomi o dei segni indicativi di un disturbo psichiatrico era del $33 \%$ nelle donne con una storia di abuso sessuale da adulte, del 15\% nelle donne con una storia di violenza fisica da parte del partner e del 6\% nelle donne che non avevano subito abusi.

- La violenza sessuale da parte di un partner amplifica gli effetti della violenza fisica sulla salute mentale.

- Il disturbo da stress post-traumatico dopo uno stupro è più frequente nel caso di ferite durante l'aggressione, o in presenza di depressione o di abuso di alcool.

- Dopo uno stupro si possono osservare anche conseguenti difficoltà nel sonno, sintomi depressivi, disturbi somatici, fumo e problemi comportamentali (quali comportamento aggressivo, furto e marinare la scuola).

È stato inoltre rilevato che:

- Le donne vittime di aggressione sessuale durante l'infanzia o l'età adulta hanno una probabilità di 3-4 volte maggiore di tentare o commettere suicidio rispetto alle altre donne.

- L'esperienza di uno stupro o di un'aggressione sessuale può determinare un comportamento suicida già nell'adolescenza.

- Anche l'esperienza di gravi molestie sessuali può determinare disturbi emotivi e un comportamento suicida. Uno studio condotto su ragazze adolescenti in Canada ha osservato come il $15 \%$ delle adolescenti vittime di contatti sessuali frequenti e non desiderati avesse manifestato un comportamento suicida nei sei mesi precedenti, rispetto al $2 \%$ delle adolescenti che non li avevano subiti.

\section{Strumentalizzazione delle vittime da parte dei mass media}

I metodi utilizzati dai mass media per discutere e rappresentare le violenze sessuali sono esaminati come fonti primarie per la perpetuazione della cultura dello stupro, e vengono spesso utilizzate come fonti che finiscono con l'influire sui risultati dei vari processi reali nei casi di violenza sessuale. Il modo in cui i media ritraggono le aggressioni sessuali può portare ad effetti dannosi sul modo in cui gli individui interpretano la violenza sessuale e le vittime della stessa. Molto spesso, infatti, i film e le pubblicità rafforzano i miti sullo stupro e finiscono con il banalizzare l'aggressione sessuale riducendola a una mera esperienza piacevole - se non persino romantica - per la vittima. È importante che i media riconoscano l'impatto che la loro narrazione ha sulla percezione di tale fenomeno nella società perché gli strumenti per un racconto corretto della violenza esistono, anche se spesso vengono ignorati. Esiste ad esempio il Manifesto di Venezia per la parità di genere nell'informazione, o ci si può avvalere del documento della Federazione Internazionale dei Giornalisti (IFJ), adottato anche dall'Ordine Nazionale dei Giornalisti, relativo a come parlare di violenza sulle donne sui media. Dal $1^{\circ}$ gennaio 2021 poi nel "Testo unico dei doveri del giornalista" è stato inserito il "rispetto delle differenze di genere", attraverso l'entrata in vigore dell'art. 5bis che riguarda il comportamento da tenere nei casi di femminicidio, violenza, molestie, discriminazioni e fatti di cronaca che coinvolgono aspetti legati all'orientamento e all'identità sessuale. Il diritto di cronaca non può trasformarsi in un abuso. "Ogni giornalista è tenuto al "rispetto della verità sostanziale dei fatti" (Manifesto di Venezia). Secondo Neate esistono principalmente due trappole insidiose per i giornalisti che affrontano tali temi: colpevolizzare le donne per quello che è successo o disumanizzarle. Spesso quando si coprono casi di violenza domestica capita di parlare con vicini o conoscenti meravigliati e ascoltare frasi come "non avremmo mai immaginato che lui potesse fare qualcosa del genere, lo conosciamo da anni ed era un marito e padre modello...".

Se si decide di costruire il proprio resoconto attorno a questo tipo di testimonianze, si sta lasciando la vittima fuori dalla storia. La donna in questi casi viene disumanizzata, trattata come oggetto accessorio di un articolo che vede come protagonista un "padre modello" o "marito esemplare" che, "non si sa perché", ha fatto qualcosa di "impensabile". Altre volte si colpevolizza la donna, concentrandosi su alcuni aspetti negativi e suggerendo in maniera non troppo sottile che parte della responsabilità sia sua, inserendo elementi che giustificano gli uomini autori di violenza: "Com'era vestita?", “Cosa aveva bevuto?", "Era attraente?", "Con quanti uomini è stata vista parlare quella notte?", "Quanti fidanzati aveva avuto finora?", "Era fedele al marito?", etc.

Quando le storie vengono distorte la donna è vittima due volte, ma la distorsione non è causata solamente da perplessità e giudizi espressi in maniera trasparente. Spesso è sufficiente l'uso di termini ambigui, contraddittori o sbagliati per alimentare una narrazione contraria ai fatti, che invece di attenersi alla realtà rincorre una sorta di romanticismo dai riscontri negativi inaspettati.

\section{Victim blaming come fenomeno sociale}

Il victim blaming non si limita quindi a rendere la violenza accettabile agli occhi di chi vi assiste, ma contribuisce ad alimentare una cultura che giustifica e supporta le dinamiche che la favoriscono. Le classiche disquisizioni mediatiche sull'abbigliamento, il comportamento o il livello di alcol nel sangue delle vittime sono rassicuranti perché, in fondo, se sei incappata in dinamiche di abuso è perché non hai rispettato le regole. Il victim blaming e lo slut shaming diventano così un fenomeno sociale di non poca rilevanza, anche in contesti sociali "culturalmente evoluti" e ciò anche per effetto della doppia morale ovvero del diverso peso che, proprio a livello sociale, determinati comportamenti assumono a seconda che vengano tenuti da uomini o donne. 
Quanto detto è tanto più vero se legato ai crimini sessuali poiché è proprio in tale ambito che il giudizio morale assume valenze diverse a seconda del genere della vittima.

I fenomeni del victim blaming e dello slut shaming hanno dato luce a diversi movimenti sociali e a rilevanti iniziative, con lo scopo di sensibilizzare le persone in merito a tali tematiche. Grande rilevanza e successo ha avuto, ad esempio, l'iniziativa promossa dagli studenti del Centro prevenzione e formazione sessuale dell'Università del Kansas, negli Stati Uniti. Gli studenti hanno incontrato alcune vittime di stupro e hanno ascoltato le loro storie e, sulla base dei loro racconti, hanno successivamente allestito la mostra dal titolo "What Were You Wearing?". Gli studenti hanno messo in esposizione 18 outfit affiancati da un panello che, in poche righe, sintetizzavano la vera storia di quelle donne, ragazze e bambine abusate. Sono stati esposti jeans, tshirt, abiti sportivi e vestiti eleganti: abiti, insomma, che testimoniavano una "vita normale". Questa mostra, in modo semplice su uno degli aspetti fondamentali quando trattiamo tale tema: non è l'abito quello che conta quando si è dinanzi a un caso di stupro. Quando si verifica un episodio così drammatico tutta l'attenzione dovrebbe concentrarsi sul carnefice, non sugli atteggiamenti o sugli abiti della vittima. Una persona violata resta in ogni caso una vittima, che non deve essere giudicata o ulteriormente privata della sua dignità e libertà.

Altro movimento che ha avuto non poca rilevanza da un punto di vista sociologico del fenomeno è quello del "This is not consent": movimento nato a seguito di un processo per stupro che ha sconvolto la città di Cork (Irlanda). Il processo, che vedeva come accusato un ragazzo di 27 anni e come vittima una ragazza diciasettenne, si risolse con l'assoluzione di lui a seguito dell'intervento dell'avvocato difensore (donna per giunta) che affermò: "Le prove escludono forse la possibilità che (la vittima) non fosse attratta dall'imputato e che non era aperta all'incontro e allo stare con qualcuno? Bisogna guardare il modo in cui era vestita. Indossava un perizoma di pizzo" (L'avvocato della difesa Elizabeth O'Connell). L'avvocato ha dunque utilizzato l'indumento intimo come prova del fatto che la vittima, ancor prima del momento dell'aggressione, fosse aperta alla possibilità di avere rapporti sessuali con qualcuno quella sera stessa. Ed è proprio a seguito dell'argomentazione della difesa, la quale non è passata inosservata alle orecchie dei cittadini, che è partito tale movimento sociale il quale, ha visto scendere in piazza a protestare migliaia di donne e uomini. La protesta è giunta fino al parlamento irlandese, luogo in cui la deputata socialista Ruth Coppinger, ha riportato all'attenzione dei colleghi l'importanza di questo caso e ha "osato" mostrare un tanga come promemoria di quanto sia avvilente, per una donna vittima di violenza, che la propria biancheria intima venga usata come prova - di un consenso mai dato - in un'aula di tribunale.

Di non meno rilevanza risulta essere anche il movimento sociale "ME TOO". Questo risulta essere un movimento di stampo femminista, che ha come obiettivo principale la lotta contro le molestie sessuali e la violenza sulle donne. Inizia a prendere piede a partire dall'ottobre 2017, anno in cui l'hashtag \#metoo inizia ad essere usato sui vari social media per dimostrare la diffusione di varie violenze sessuali e molestie subite dalle donne, specie sui luoghi di lavoro e studio. Galeotto di tale movimento è stato il diffondersi delle rivelazioni pubbliche di accuse di stupro contro Harvey Weistein. Quello del Me Too risulta essere un movimento importante che ha portato alla luce violenze e abusi che altrimenti non sarebbero stati denunciati; ciononostante vi sono stati anche dei risvolti negativi a seguito di tale movimento in quanto, basandosi su questo semplice hashtag, l'opinione pubblica ha finito con il giudicare colpevoli a priori tutti gli accusati di molestie senza prove effettive raccolte dalle autorità competenti. È dunque impor- tante credere a una vittima quando questa sporge una denuncia a seguito di un'aggressione, ma altrettanto importante è svolgere le indagini necessarie per stabilire se tale fatto sia stato messo in atto o meno, sempre nel rispetto della vittima, della sua condizione psicofisica ma anche nel rispetto dell'indagato stesso.

È fondamentale portare la società ad eliminare fenomeni quali quello del victim blaming e dello slut shaming, è fondamentale costruire una società in cui le vittime non abbiano paura di essere giudicate a seguito di una violenza subita, ma bisogna anche stare attenti a non finire con l'appesantire troppo l'altro piatto della bilancia.

Il movimento Me Too è stato fondamentale per far prendere coscienza all'opinione pubblica (o quanto meno a parte di essa) di quanto sia difficile per una vittima di violenza sessuale denunciare e affrontare tutto il processo che segue quella denuncia. Scopo di questo movimento è stato quello di rendere la società meno rigida nei confronti delle vittime di violenza sessuale obiettivo ancora da dover raggiungere - ma non deve diventare un pretesto per supporre a priori la colpevolezza di ogni persona che viene accusata. Per quanto la società abbia bisogno di credere in un "mondo giusto", non sono rare le false denunce di violenza sessuale messe in atto da donne. Se iniziamo a sostenere un processo basato su qualcuno che si fa avanti con un'accusa e si ignora il processo investigativo, tutto ciò che il movimento Me Too aveva il potenziale di risolvere, verrà interrotto e spostato nella direzione opposta.

\section{Alcuni dati statistici}

In Italia ogni anno si denunciano circa 4.000 violenze sessuali (Fig. 1). Secondo gli esperti questo numero non riflette la realtà: gli episodi di violenza sono molti di più. Secondo l'Istat nel $92,5 \%$ dei casi le donne che hanno subito una violenza sessuale non l'ha denunciata (la percentuale sale al 95,6\% se l'aggressore è italiano e scende al $75,3 \%$ se è straniero), e da questo conto sono escluse le violenze commesse dai partner e dagli ex partner. Il baratro nel quale queste donne sono catapultate chiama in causa l'intero sistema che dovrebbe proteggerle e di cui, evidentemente, non si fidano. È un silenzio solido e dalle dimensioni spaventose: secondo lo studio 652.000 donne tra i 16 e i 70 anni sono state vittime della violenza più brutale, lo stupro, e 746.000 di tentativi falliti ma non per questo senza conseguenze fisiche e psicologiche.

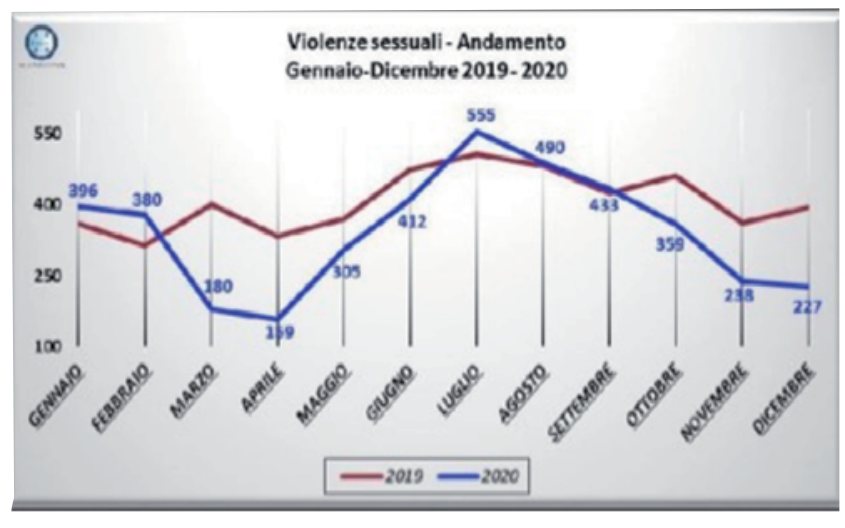

Fig. 1. Violenze sessuali nel 2019-2020. Fonte: Ministero dell'Interno, direzione centrale della polizia criminale. 
Un capitolo a parte, che spiega forse meglio di altri gli automatismi che non fanno scattare la denuncia, è la molestia sessuale al lavoro. In questo luogo lontano dalle dinamiche di coppia, il $7,5 \%$ delle donne dice di aver subito almeno un ricatto sessuale. L' $80,9 \%$ non ne ha parlato con nessuno, solo lo $0,5 \%$ ha denunciato il molestatore. E poi? E poi una donna su tre ha cambiato lavoro o ha rinunciato volontariamente alla carriera $(34 \%)$, più di una su dieci è stata licenziata (11\%) e solo nel 6,5\% dei casi è andato via il molestatore. In un caso su cinque non è successo assolutamente nulla.

Un importante tutela alle vittime di stupro, e più in generale ad ogni vittima di violenza, viene garantita, nel nostro paese, della legge 69/2019 ovvero il famoso Codice Rosso. Nonostante la legge 69/2019 abbia come scopo ultimo quello di tutelare le vittime, queste ultime si vedono sempre più scoraggiate a denunciare quanto successo loro a causa della non certezza della pena cui andrà in contro il loro aggressore, la lunghezza dei processi e l'alto numero di archiviazioni di casi (spesso dovuti alle inadempienze dei tribunali e a un sistema giuridico e burocratico che, nel suo insieme, non tutela la donna e i suoi diritti). I servizi più frequenti offerti alle vittime di violenza sessuale, da parte dei centri antiviolenza, sono: ascolto e accoglienza, orientamento e accompagnamento ad altri servizi della rete territoriale $(96,5 \%)$, supporto legale $(93,8 \%)$, supporto e consulenza psicologica $(92,2 \%)$, sostegno all'autonomia $(87,5 \%)$, percorso di allontanamento $(84,0 \%)$ e orientamento lavorativo $(80,5 \%)$.

Secondo dati dell'Agenzia per i diritti fondamentali dell'Unione europea, i Paesi in cui la violenza contro le donne (fisica e/o sessuale) è più comune sono quelli del Nord Europa. In Finlandia, ogni anno 50.000 donne subiscono violenza sessuale, compreso lo stupro. La maggior parte dei responsabili non sono portati di fronte alla giustizia: nel 2017 vi sono state solo 209 condanne per stupro. Per quanto riguarda la Svezia, nel 2017 sono andati in giudizio solo il $6 \%$ dei casi in cui erano coinvolte persone adulte. In Danimarca va persino peggio: su 24.000 donne che nel 2017 hanno subito uno stupro o un tentato stupro, solo 890 casi sono stati denunciati alla polizia: di questi, 535 sono arrivati a un processo e le condanne sono state solo 94.

Dall'analisi dell'Agenzia emerge che i Paesi in cui le donne raccontano maggiormente casi di violenza sono i Paesi in cui la violenza sessuale risulta essere percepita come meno comune. A questo proposito, in Italia il $90 \%$ delle donne pensano che la violenza sessuale sia un problema dilagante, anche se gli episodi rilevati non arrivino al 20\%. Stessa cosa in Portogallo, dove il 93\% delle donne pensa che la violenza sessuale sia piuttosto comune, sebbene le testimonianze delle donne che denunciano di aver subito "attenzioni sessuali" rappresenti il 15\% dei casi. In Finlandia, si verifica invece il fenomeno opposto: meno del $70 \%$ delle donne ritiene la violenza sessuale un problema comune, a fronte di una situazione di gran lunga sopra la media europea.

In Norvegia, Finlandia e Danimarca, stando ad un rapporto di Amnesty International del 2019, un fattore chiave nel negare l'accesso alla giustizia alle vittime di stupro è la legge stessa. Sebbene tutti e tre i paesi siano parte della Convenzione del Consiglio d'Europa sulla prevenzione e la lotta alla violenza contro le donne e la violenza domestica (Convenzione di Istanbul), devono ancora introdurre una legislazione che definisca chiaramente lo stupro in termini di mancanza di consenso. Mentre le definizioni nelle leggi di ogni paese variano, ognuna - in modi diversi - definisce lo stupro in termini di uso o minaccia di violenza o capacità della vittima di resistere, piuttosto che in assenza di un consenso liberamente dato. Ciò ha enormi ripercussioni sia in termini di capacità di perseguire con successo i crimini, ma anche in termini di come la società stessa percepisce lo stupro.

\section{L'uomo vittima di violenza sessuale}

Facendo un'analisi approfondita dei reati a matrice sessuale emerge come, a causa di radicati pregiudizi sociali, spesso si è portati ad ignorare la figura maschile nel suo ruolo di vittima. La presenza di comportamenti fisicamente e/o psicologicamente violenti, messi in atto da entrambi i partner, nelle coppie è un fenomeno diffuso e trasversale, indipendentemente da fattori quali il contesto sociale, le etnie o le culture di appartenenza. La violenza sessuale contro le donne è tanto diffusa quanto quella nei confronti degli uomini, eppure solo di recente sono state avviate delle ricerche in questo ambito.

Ad oggi non esiste una definizione, riconosciuta a livello internazionale, di violenza specifica contro gli uomini. Un'importante differenza tra uomo e donna risiede nella percezione del maltrattamento, alcune forme di violenza sono considerate talmente "normali" dagli uomini da non essere neppure prese in considerazione. Si tratta per esempio di atti di violenza fisica subiti in pubblico, come le risse, considerate alla stregua di semplici diverbi. Altre forme di violenza sono tabù, al punto da spingere coloro che le subiscono a non parlarne o a rimuoverle. Questo vale specialmente per la violenza di carattere sessuale contro gli uomini. Spesso gli uomini maltrattati in famiglia si vergognano di ammetterlo e non osano parlarne nemmeno con i familiari più stretti o con gli amici più intimi. Essi devono tuttavia temere più delle donne di non essere presi sul serio e questo li porta a non ammettere di essere vittime, a non riconoscersi in questa immagine che a loro parere non si concilia con l'immagine di "vero uomo".

Importante risulta l'indagine conoscitiva sulla violenza verso il sesso maschile condotta da Macri, Loha e Gallino nel 2012, che ha come scopo ultimo della ricerca quello di verificare se, in accordo con la letteratura scientifica internazionale anche la popolazione maschile italiana può essere vittima di violenza fisica-sessuale-psicologica da parte del partner o ex partner di genere femminile. Da quanto emerso dall'indagine, condotta su un totale di 1058 soggetti, per quanto riguarda la violenza fisica il $63,1 \%$ del campione dichiara di aver subito almeno un episodio per mano di una donna nel corso della propria vita; per la violenza sessuale risulta essersi verificato nel $48,7 \%$ soggetti e per la violenza psicologica nel $77,2 \%$; per quanto riguarda gli atti persecutori il 31,9\% del campione ha subito almeno un episodio. Riguardo alla violenza sessuale il $4,1 \%$ dei soggetti intervistati ha dichiarato di essere stato forzato ad avere rapporti sessuali con altre persone incluso sesso di gruppo o scambi di coppia. La maggiore rappresentatività del campione è compresa nella fascia d'età 40-49, seguita dalla fascia di età 30-39. Lo stato civile maggiormente presente è quello dei separati $(41,1 \%)$, seguito dai celibi $(24,3 \%)$. Le più recenti stime degli Stati Uniti, indicano che il $15,2 \%$ di coloro che hanno subito violenza sessuale nella loro vita, sono uomini. I Centers for Disease Control and Prevention e il National Institute of Justice hanno rilevato che, 92.700 uomini adulti sono violentati ogni anno negli Stati Uniti e circa il 3\% di tutti gli americani uomini, per un totale di 2,78 milioni di uomini, hanno sperimentato un tentativo di stupro o violenza compiuta nella loro vita (Basile, 2007). In uno studio condotto in Inghilterra, si evidenzia un tasso di prevalenza del $2,89 \%$ rispetto alla violenza sessuale. Inoltre, in Inghilterra e Galles, lo stupro maschile comprende il 7,5\% di tutti gli stupri denunciati.

L'Organizzazione Mondiale della Sanità afferma che tra il 5\% e il $10 \%$ degli uomini, in tutto il mondo, riportano una storia di abuso sessuale infantile. Altre stime internazionali dell'infanzia indicano che tra il 3\% e il $29 \%$ dei maschi hanno subito forme di violenza sessuale. 
I pregiudizi nei confronti degli uomini vittime di violenza sono evidenti dal fatto che, la società stessa, ha uno scarso numero di servizi di supporto forniti a questa tipologia di vittime. I vari supporti, e le varie risorse, messe a disposizione delle vittime donne, non sono messe anche a disposizione delle vittime di sesso maschile e, nei rari casi in cui queste ultime ricevano assistenza, i professionisti e le professioniste con cui entrano in contatto dimostrano una forte lacuna in merito alle giuste metodologie da utilizzare per garantire un supporto e un sostegno adeguato alle vittime maschili.

\section{La legge 15 febbraio 1996 n. 66 e il concetto di consenso}

A partire dalla fine degli anni Settanta, il tema dei reati in materia sessuale è tornato ad essere oggetto di numerose proposte di legge, volte a riformulare più o meno integralmente le fattispecie disciplinate dal Codice Rocco. A seguito della famosa vicenda del Circeo, che suscitò, all'epoca, grande clamore nell'opinione pubblica, nel 1995 venne approvata dalla Camera la proposta n. 2576 presentata il 23 maggio 1995 che, passata all'esame del Senato, divenne la Legge 15 febbraio 1996 n. 66 "Norme contro la violenza sessuale". Tale legge ha abrogato, con 1'art.1, il Capo I del Titolo IX del Libro II ed introducendo, con l'art. 2, i delitti contro la libertà personale. Grande innovazione si è avuta specie da un punto di vista culturale e simbolico in quanto si è attuata una riqualificazione dei delitti sessuali come "delitti contro la persona", riqualificazione auspicata da decenni e condivisa da tutti, perché conforme a quella concezione personalistica della tutela, fatta propria anche dalla Costituzione repubblicana e che aveva reso intollerabilmente antinomia e anacronistica la qualificazione autoritario-pubblicistica dei delitti sessuali come delitti contro la moralità pubblica e il buon costume. La legge ha portato all'introduzione di articoli (da 609 bis a 609 decies) volti a colmare quegli spazi vuoti lasciati liberi dalle precedenti legislazioni. Importante è anche sottolineare l'unificazione delle fattispecie di "violenza carnale" (ex art. 519) e "atti di libidine violenti" (ex art. 521) nel reato di violenza sessuale (art. 609 bis c.p.), l'introduzione della fattispecie della violenza sessuale di gruppo, prevista dall'art. 609-octies c.p. (anche se è doveroso rammentare che si è parecchio discusso in ordine alla natura giuridica dell'istituto che secondo taluni è una figura autonoma di reato, secondo altri una circostanza aggravante della figura base di violenza sessuale individuale), l'introduzione di una fattispecie autonoma (art. 609 quater c.p.) che incrimina il compimento di atti sessuali, violenti o abusivi, nei confronti dei minori, la previsione della non punibilità dei rapporti sessuali tra minorenni, la modificazione del delitto di corruzione di minorenne, l'aumento di due anni del minimo di pena (reclusione da 5 a 10 anni anziché da 3 a 10 anni per il reato circostanziato di violenza sessuale).

Nello specifico, l'art. 609-bis c.p. prevede che: «Chiunque, con violenza o minaccia o mediante abuso di autorità, costringe taluno a compiere o subire atti sessuali è punito con la reclusione da sei a dodici anni. Alla stessa pena soggiace chi induce taluno a compiere o subire atti sessuali:

1. abusando delle condizioni di inferiorità fisica o psichica della persona offesa al momento del fatto;

2. traendo in inganno la persona offesa per essersi il colpevole sostituito ad altra persona.

Nei casi di minore gravità la pena è diminuita in misura non eccedente i due terzi (Brocardi). Risulta evidente come, in termini giuridici, si tratti di un reato comune, potendosi attribuire la qualifica di soggetto attivo a "chiunque". Fulcro della condotta criminosa diventa così l'atto sessuale, nozione che unifica, come prece- dentemente affermato, le espressioni anteriforma di "congiunzione carnale" ed "atti di libidine violenti". La riforma mirava a risolvere, in tal modo, problemi legati alla qualificazione giuridica delle condotte che comportavano, sovente, una sottoposizione della vittima a domande mortificanti ed invasive della sfera intima e della privacy, ai fini dell'accertamento del reato commesso. È stato poi osservato, in realtà, che l'unificazione non escludeva la possibilità che simili domande fossero poste ai fini della commisurazione della pena.

Nel 2006 la Cassazione che affermato che il reato di violenza sessuale è configurabile in presenza di "ogni forma di costringimento fisico-psichico, idonea in qualche modo ad incidere sull'altrui libertà di autodeterminazione, se finalizzata al compimento di un atto sessuale, [...] anche all'interno del rapporto di coppia, coniugale o para coniugale che sia", aggiungendo che: "è pur sempre necessario che l'agente abbia consapevolezza della condizione della vittima e del rifiuto in essa implicito all'atto sessuale. Un rifiuto che può seguire ad un'originaria adesione e collocarsi in ogni momento del rapporto sessuale, rendendo assoggettabile a sanzione il segmento dell'altrui condotta che si protragga contro la volontà dell'avente diritto" (Cass. pen., Sez. III, 12 maggio 2006, n. 16292).

Aspetto fondamentale, dunque, viene dato anche al concetto di consenso da parte della vittima. La mancanza di consenso è elemento costitutivo del reato di violenza sessuale e, quindi, in presenza di esso non vi è, per definizione, tipicità del fatto. Da un punto di vista giuridico, il consenso è un permesso che si da a qualcun altro di agire ed implica un accordo caratterizzato da:

1) comprensione di ciò che è stato proposto;

2) conoscenza degli standard sociali relativi a ciò che è stato proposto;

3) consapevolezza delle conseguenze e delle alternative;

4) la sicurezza che ogni decisione verrà rispettata;

5) volontarietà della decisione.

Esso è giuridicamente rilevante solo se valide, ovvero se è espresso da qualcuno che dispone del diritto di dare un consenso, se questa persona ne ha le capacità ed è libera di agire (Fornari, 2008). È, inoltre, fondamentale sottolineare che il consenso è valido non solo se lo si è pienamente manifestato in fase iniziale, ma anche se non lo si è revocato e se questo non è stato condizionato. Stando alla sentenza della Cassazione penale, sez. III, del 20 novembre 2019 (sentenza n.10372) "Ai fini della sussistenza dell'elemento soggettivo del reato di violenza sessuale è sufficiente che l'agente abbia la consapevolezza del fatto che non sia stato chiaramente manifestato il consenso da parte del soggetto passivo al compimento degli atti sessuali a suo carico, cosicché integra il reato non soltanto la condotta invasiva della sfera della libertà e integrità sessuale altrui realizzata in presenza di una manifestazione di dissenso della vittima, ma anche quella posta in essere in assenza del consenso, non espresso neppure in forma tacita, della persona offesa, come nel caso in cui la stessa non abbia consapevolezza della materialità degli atti compiuti sulla sua persona". Dunque, per la sussistenza dell'elemento soggettivo non è necessario l'espresso dissenso, ma basta l'assenza del consenso della vittima. La giurisprudenza ha optato per un modello che fa leva sul concetto di violenza incentrato sul vulnus della volontà del soggetto passivo piuttosto che sull'esercizio di una forza fisica strumentale agli atti sessuali.

Dando uno sguardo alle diverse legislazioni internazionali emerge che:

- La legislazione francese stabilisce che l'espressione del consenso è valida solo se non sono state esercitate violenza, pressioni fisiche o psicologiche; l'agente non deve aver abusato della sua posizione di superiorità o minacciato rappresaglie in 
caso di rifiuto e che la vittima non fosse incosciente o sotto effetto di alcol o droghe. A differenza della legge italiana, non esiste un'età minima per il consenso. Nello specifico, in Italia, un maggiorenne può compiere atti sessuali consenzienti con minori, solo se questi hanno compiuto 14 anni (art. 609-quater, comma 1). Se il maggiorenne è un ascendente, il genitore, anche adottivo, o il di lui convivente, il tutore, ovvero altra persona cui, per ragioni di cura, di educazione, di istruzione, di vigilanza o di custodia, il minore è affidato o che abbia, con quest'ultimo, una relazione di convivenza, allora può compiere atti sessuali solo con minorenni consenzienti, di 16 anni compiuti (art. 609-quater c.p., comma 1). Questi adulti, se ottengono il consenso del maggiore di 16 anni con l'abuso dei poteri connessi alla loro posizione, commettono un reato (art. 609quater c.p., comma 2). I minorenni possono avere rapporti consenzienti a partire dal compimento dei 13 anni, purché l'altro sia un minore con una differenza massima d'età di 4 anni. (art. 609-quater c.p., comma 4). Il reato è punibile a querela della persona offesa (art. 609-septies c.p.), ma è procedibile d'ufficio nel caso in cui:

a) il minore abbia meno di dieci anni;

b) il fatto è connesso con un altro delitto per il quale si deve procedere d'ufficio;

c) il fatto è commesso dall'ascendente, dal genitore, anche adottivo, o dal di lui convivente, dal tutore ovvero da altra persona cui il minore è affidato per ragioni di cura, di educazione, di istruzione, di vigilanza o di custodia o che abbia con esso una relazione di convivenza;

d) il fatto è commesso da un pubblico ufficiale o da un incaricato di pubblico servizio nell'esercizio delle proprie funzioni. Nel caso in cui gli atti sessuali avvengano consenzientemente in cambio di denaro o altra utilità economica con un minore di 18 anni, anche se maggiore dell'età del consenso, si ha il reato di prostituzione minorile (art. 600bis, comma 2 c.p.). In merito alla possibilità di un eventuale errore relativamente all'età del minore di anni 18 l'articolo '609-sexies' del codice penale specifica che: “Quando i delitti previsti negli articoli 609-bis, 609-ter, 609-quater, 609-octies e 609-undecies sono commessi in danno di un minore degli anni diciotto, e quando è commesso il delitto di cui all'articolo 609-quinquies, il colpevole non può invocare a propria scusa l'ignoranza dell'età della persona offesa, salvo che si tratti di ignoranza inevitabile" (articolo 609-sexies);

- La legislazione tedesca, invece, solo recentemente ha modificato la normativa sulla violenza sessuale che prevedeva, all'art.177 del c.p., che per parlare di reato sessuale la vittima dovesse dimostrare di essersi difesa fisicamente, non essendo sufficiente il rifiuto verbale. Con la modifica si è riconosciuto valore anche al dissenso manifestato verbalmente.

- Negli Stati Uniti, con la legge del 2014 viene introdotto il concetto di "consenso affermativo, cosciente e volontario", il quale viene richiesto per qualsiasi forma di interazione sessuale e, per ogni atto sessuale che non abbia tale tipo di consenso viene considerato a tutti gli effetti una violenza. Pertanto, viene considerata violenza sessuale ogni rapporto messo in atto con una persona incapace di esprime il suo pieno assenso.

\section{Conclusioni}

Si parla poco di vittime, di coloro che sono stati sottoposti ad eventi tanto drammatici da portarne a lungo gli esiti fisici, psicolo- gici e morali, dei loro bisogni e dei loro diritti. In genere l'attenzione si concentra sull'autore del reato e sulle cause dell'evento critico, mentre l'interesse per la vittima tende rapidamente a scemare. Nonostante i progressi culturali e scientifici intorno ai temi vittimologici, nel nostro tempo, le istituzioni, l'ambiente sociale e l'opinione pubblica continuano ad assumere atteggiamenti di negazione, di inadeguato riconoscimento e di condanna morale nei confronti della vittima. Per anni le vittime sono state colpevolizzate. Lo stigma della vittima "colpevole quanto il carnefice" introdotta dai primi studi vittimologici, richiamando la deresponsabilizzazione del reo, ancora oggi fa parte dei pregiudizi dell'immaginario collettivo. Quanto emerge dall'analisi effettuata in tale articolo è che, siamo di fronte a un fenomeno complesso e ben radicato a livello sociale, la cui spiegazione non può essere semplicisticamente ricondotta a un unico antecedente, ma deve tenere conto di molteplici fattori come il clima socioculturale e l'interiorizzazione di valori - conservatori - da parte delle persone di false credenze circa la violenza stessa. È emerso come, l'ingiustizia strutturale nei confronti delle donne, è perpetrata dalle pratiche di colpevolizzazione delle vittime, che vanno così a limitare la libertà di espressione e la fruizione dei diritti del gruppo a cui le vittime appartengono. Per questa ragione, l'ascolto empatico dell'altro permette di stabilire un contatto più approfondito e si rivela terapeutico, andando ad interrompere l'isolamento provocato dalla violenza. La sensibilizzazione e la formazione del personale impiegato in sede investigativa appaiono quindi fondamentali. L'approccio alla violenza sessuale dovrebbe mutare, ripensando quei concetti giuridici e culturali che vedono la donna come responsabile di quanto le è accaduto a causa del suo modo di vestire, della sua decisione di non rincasare a orari socialmente concepiti come "consoni", contribuendo allo stigma sociale che rende difficile l'accertamento processuale. I fenomeni del victim blaming e dello slut shaming colpiscono principalmente le donne che non si conformano ai modelli mainstream di femminilità. Le pratiche di colpevolizzazione sono volte a rinforzare le asimmetrie di potere tra vittime e oppressori, incentivando quei modelli di comportamento che non prevedono forme di reazione all'oppressione. Si dovrebbe mirare a disinnescare i dispositivi culturali che considerano le vittime responsabili per ciò che accade loro, attraverso programmi di sensibilizzazione che contribuiscono allo sviluppo di un atteggiamento critico.

Rispetto a possibili ricerche future, un aspetto importante, ancora oggi poco approfondito, riguarda le conseguenze di essere oggetto di biasimo da parte di osservatori esterni. Da un punto di vista più ampio, emerge la necessità di sviluppare programmi che non solo mirino alla prevenzione della violenza di genere, ma che disinneschino anche i meccanismi legati al victim blaming. Vi è, infatti, la necessità di rendere consapevoli le persone del fatto che molti dei giudizi emessi "con spontaneità" o "con buon senso" sono influenzati invece da false credenze socialmente diffuse. I programmi di sensibilizzazione dovrebbero essere costruiti in modo tale da mettere le persone in una condizione di consapevolezza, permettendo loro di sviluppare un atteggiamento critico verso questi dispositivi culturali così da disinnescare questi automatismi che portano a considerare le vittime responsabili per ciò che è accaduto loro. Per fare ciò si dovrebbe agire su più livelli: da un lato i media dovrebbero parlare della violenza di genere senza legittimare e rafforzare queste false credenze. Dall'altro, dovrebbero essere creati dei programmi e degli interventi di sensibilizzazione, non solo rivolti alle professioniste e ai professionisti che a vario titolo entrano in contatto con le vittime di violenza di genere, ma rivolti anche alla popolazione in generale a partire già dall'infanzia così da ostacolare la precoce interiorizzazione di queste credenze stereotipiche. 


\section{Bibliografia}

Di Stefano A (2012). Violenza contro le donne e violenza domestica nella nuova Convenzione del Consiglio d'Europa. Diritti umani e diritto internazionale 6:169-176.

Ainsworth MDS, Blehar M, Waters E, Wall S (1978). Patterns of attachment: assessed in the strange situation and at home. Hillsdale: Erlbaum.

Ambrosini G (1997). Le nuove norme sulla violenza sessuale. Torino: UTET.

American Psychiatric Association (1994). Diagnostic and statistical manual of mental disorders (DSM-V). Washington: APA Press, 1994 (trad. it. Milano, Masson, 1995).

Antolisei F (1996). Manuale di diritto penale. Parte speciale, I. Milano: Giuffrè Editore.

American Psychiatric Association (2014). DSM-V. Manuale Diagnostico e statistico dei disturbi mentali. MilanoM: Raffaello Cortina Editore.

Balbi G. Violenza sessuale, op. cit., 22.

Bard M, Sangrey D. (1986). The crime victim's book. New York: Lyle Stuart.

Basile KC, et al. (2007). Prevalence and characteristics of sexual violence victimization among US adults, 2001-2003. Violence Vict 22:437-441.

Beauregard E, Mieczkowski T (2012)."Risk estimations of the conjunction of victim and crime event characteristics on the lethal outcome of sexual assaults. Violence Vict 27:470-486.

Beltrani M. Le nuove norme sulla violenza sessuale. op. cit., 65 ss

Benedict H (1992). Virgin or vamp: How the press covers sex crimes". New York: Oxford University Press.

Berti C (2002). Psicologia sociale della giustizia. Bologna: Il Mulino.

Bertolino M., Libertà sessuale e tutela penale., op. cit., 1993, $139 \mathrm{ss}$

Brownmiller S (1975). Against our will: Men, women, and rape". New York: Fawcett Books.

Bruzzone R, Caputo A (2019). Criminologia dei sex offender. Milano: Giuffrè Editore.

Burgess AW, Hartman CR, Ressler RK, Douglas JE, McCormack A, op. cit., 266.

Burgess AW, Hartman CR, Ressler RK, Douglas JE, McCormack A, op. cit., 246.

Cook P (2009). Abused men. The hidden side of domestic violence. Westport: Praeger.

Cooper RW (1995). Sesso estremo. Roma: Castelvecchi.

Corte Costituzionale, ordinanza 11 luglio 2000, n. 295

Corte Europea dei Diritti Umani, Prima Sezione, 27 maggio 2021, J.L. c. Italia, n. 5671/16, § 141.

Degani P (2000). Donne, diritti umani e conflitti armati. La questione della violenza nell'agenda della comunità internazionale. Research paper. Centro diritti umani - Università di Padova.

Dèttore D, Fuligni C (1999). L'abuso sessuale sui minori. Valutazione e terapia delle vittime e dei responsabili. Milano: McGraw-Hill.

Eher R, Rettenberger M, Turner D (2019). The prevalence of mental disorders in incarcerated contact sexual offenders. Acta Psychiatr Scand 139:572+581.

Fiandca, voce Violenza sessuale, in Enciclopedia del Diritto (Agg. IV), Milano, 2000, 1158.

Finkelhor D, Turner H, Ormrod R, et al. (2009). Children's exposure to violence: A comprehensive national survey. NCJ 227744. Department of Justice, Office of Justice Programs, Office of
Juvenile Justice and Delinquency Prevention. https://www.ncjrs. gov/pdffiles1/ojjdp/227744.pdf

Fischer CT, Wertz FJ (1979). Empirical phenomenological analyses of being criminally victimized. In: A. Giorgi, R. Knowles, D.L. Smith (a cura di), Duquesne studies in phenomenological psychology. Pittsburgh: Duquesne University Press, pp. 135158.

Gatta GL (2009). Violenza sessuale: nuova aggravante. In: S. Corbetta, A. Della Bella, GL Gatta (a cura di), Sistema penale e sicurezza pubblica. Le riforme del 2009. Milano: Ipsoa.

Gery I, Miljkovitch R, Berthoz S, Soussignan R. (2009). Empathy and recognition of facial expressions of emotion in sex offenders, non-sex offenders and normal controls. Psychiatry Research 165:252-62

Giulini P, Xella CM (2011). Buttare la chiave? La sfida al trattamento per gli autori di reati sessuali. Milano: R. Cortina Editore.

Godson S (2003). Il libro del sesso. Milano: Sonzogno.

Hanson RK, Helmus L, Babchishin KM, Mann RE (2013). Attitudes supportive of sexual offending predict recidivism: A metaanalysis. Trauma Violence Abuse 14:34-53.

Lambert S, Hammond S (2009). Perspectives on female sexual offending in an Irish context. Irish Journal of Applied Social Studies 9:4.

Lanning KV (1992). Child molesters: a behavioral analysis. National Center for Missing and Exploited Children.

Lerner M (1980). The belief in a just world. A Fundamental Delusion. New York: Plenum Pub Corp.

Lupo E (2010). Codice penale. Rassegna di giurisprudenza e dottrina, vol. XI, tomo II, I delitti contro la persona, Milano, 478.

MacCulloch MC, Snowden PJ, Wood P, Mills HE (1983). Sadistic fantasy, sadistic behavior, and offending. British Journal of Psychiatry 143:20-29.

Macri PG, Loha YA, Gallino G, et al (2012). Rivista di Criminologia, Vittimologia e Sicurezza Vol. VI n. 3.

Mantovani F (1998). Diritto penale. Parte speciale. I delitti contro la libertà e l'intangibilità sessuale, op. cit., 72, 45.

Marani S (2014). Reati contro la donna. Excursus normativo. Roma: Altalex Editore.

Marini G (1996). Delitti contro la persona. Torino: Giappichelli Editore.

Marini G (1994). Moralità pubblica e buon costume (delitti contro la), in Dig. Pen., vol. VIII, 443.

Melchionda A, Commento all'art. 4 della Legge sulla violenza sessuale, in "Commentari delle norme contro la violenza sessuale e della legge contro la pedofilia", op. cit., 94 e 161 ss

Mieczkowski T, Beauregard E (2010). Lethal outcome in sexual assault events: A conjunctive analysis. Justice Quarterly 27: 332- 361

Moccia S. Il sistema delle circostanze e le fattispecie qualificate nella riforma del diritto penale sessuale (L. 15 febbraio 1996, n. 66): un esempio paradigmatico di sciatteria legislative, cit., 405.

Musacchio V (1997). La nuova legge sulla violenza sessuale. Riv Pen 268.

National Sexual Violence Resource Center, 2013

Padovani T (2006). Commento art. 1. In: A. Cadoppi (a cura di), Commentario delle norme contro la violenza sessuale e della legge contro la pedofilia. op. cit., 5 ss.

Palermo GB, Mastronardi VM (2005). Il profilo criminologico. Dalla scena del crimine ai profili socio-psicologici. Milano: Giuffrè Editore.

Petruccelli I, Pedata LT (2008). L'autore di reati sessuali. Valutazione, trattamento e prevenzione della recidiva. Milano: Franco Angeli. 
Polaschek DDL, Ward T (2002). The implicit theories of potential rapists: What our questionnaires tell us. Aggression and Violent Behavior 7:385-406.

Purcell CE, Arrigo BA (2006). The psychology of lust murder. Paraphilia, sexual killing, and serial homicide. Burlinghton: Academic Press, pp.53-65.

Puzzo C (2010). I reati sessuali. Milano: Maggioli Editore, p. 46.

Quattrini F (2015). Parafilie e devianza. Psicologia e psicopatologia del comportamento sessuale atipico. Firenze: Giunti Editore.

Robertiello G, Terry KJ (2007). Can we profile sex offender? A review of sex offender typologies. in and Violent Behaviour 12:508-518

Romano B (2016). Delitti contro la sfera sessuale della persona. Milano: Giuffrè Editore, p. 98.

Romano B (2000). La tutela penale della sfera sessuale. Indagine alla luce delle recenti norme contro la violenza sessuale e contro la pedofilia. Milano: Giuffrè Editore, p. 48.

Rotoli G (2012). Indagine conoscitiva sulla violenza verso il maschile. Rivista Criminologia, Vittimologia e Sicurezza VI.

Ryan W (1976). Blaming the victim. London: Vintage Books.

Sandler JC, Freeman NJ (2007). Typology of female sex offenders: A test of Vandiver and Kercher. Sexual Abuse 19:73-89.

Sigler A (2017). Risk and prevalence of personality disorders in sexual offenders. Thesis Dissertation, John Jay College of Criminal Justice.

Sigre-Leirós V, Carvalho J, Nobre P (2014). Cognitive schemas and sexual offending: Differences between rapist, pedophilic and nonpedophilic child molesters, and nonsexual offenders. Child Abuse Neglect 40:81-92.

Simonelli C, Petruccelli F, Vizzari V (2000). Le perversioni sessuali. Milano: Franco Angeli.

Smith SG, Chen J, Basile KC, et al. (2017). The National Intimate Partner and Sexual Violence Survey (NISVS): 2010-2012 State Report. Atlanta: National Center for Injury Prevention and Control, Centers for Disease Control and Prevention.

Spagnolo. La problematica dei rapporti sessuali con minori e tra minori. Riv it dir proc pen - cit., 72 .

Steinmetz S (1978). The battered husband syndrome. Victimology 2:499-509.

Stemple L (2009). Male rape and human rights. Hastings Law Journal 60:605.

Tanenbaum L (2015). I am not a slut: Slut-shaming in the age of the internet. New York: Harper Perennial.

Troiano M, Petrone L (2005). E se l'orco fosse lei? strumenti per l'analisi, la valutazione e la prevenzione dell'abuso al femminile: con nuovo test per la diagnosi. Milano: Franco Angeli

Venturioli M (2012). La tutela della vittima nelle fonti europee. www.penalecontemporaneo.it

Vessichelli. Violenza sessuale. Come cambia il codice penale. in Guida dir., cit., 24.

Zangheri Neviani C (2014). Un contributo alla riflessione sul concetto di vittima. Nuove Frontiere Diritto - Speciale n. 2: 79-86.
Correspondence: Aurelia V. Nemesu.

E-mail: nemesuaurelia26@gmail.com

Key words: victim blaming; sexual abuse; sexual violence.

Parole chiave: victim blaming; abusi sessuali; violenze sessuali. Palabras clave: culpabilización de la víctima; abuso sexual; violencia sexual.

Received for publication: 29 July 2021.

Accepted for publication: 1 September 2021.

This article is distributed under the terms of the Creative Commons Attribution Noncommercial License (by-nc 4.0) which permits any noncommercial use, distribution, and reproduction in any medium, provided the original author(s) and source are credited.

${ }^{\circ}$ Copyright: the Author(s), 2021

Licensee PAGEPress, Italy

Rivista di Psicopatologia Forense, Medicina Legale, Criminologia 2021; 26:632

doi:10.4081/psyco.2021.632 